\title{
Prevalence of glaucoma in first-degree relatives of patients with primary open-angle glaucoma and normal-tension glaucoma
}

Preeti Gupta, Vijaya Pai H.

Department of Ophthalmology, Kasturba Medical College, Manipal, Manipal Academy of Higher Education, Karnataka, India

\begin{abstract}
Objective: To determine the prevalence of glaucoma in first-degree relatives of patients with primary open-angle glaucoma (POAG) and normal tension glaucoma (NTG).

Methods: Observational study of first-degree relatives of patients with POAG and NTG who were screened for glaucoma.

Results: A total of 66 first-degree relatives of patients with glaucoma (POAG/NTG) were included in the study. We found a prevalence rate of $16.6 \%: 13.6 \%$ were diagnosed to have the disease and $3 \%$ were newly detected to have glaucoma during the study. We found that although $66.7 \%$ of the subjects were aware of the family history of glaucoma, only $36.4 \%$ were aware that they were at increased risk of developing glaucoma.

Conclusion: In our study, we found high prevalence of glaucoma in first-degree relatives of patients with glaucoma. This highlights the importance of selective screening of high-risk groups such as first-degree relatives of patients with POAG/NTG.
\end{abstract}

Keywords: awareness, first-degree relatives, glaucoma, increased risk, prevalence

\section{Introduction}

Glaucoma is the most common cause of irreversible blindness worldwide. ${ }^{1}$ In southern India, the prevalence of open-angle glaucoma is estimated to be $1.6 \%$ of the population, with more than $98 \%$ being unaware that they have the disease. ${ }^{2}$

Primary open-angle glaucoma (POAG) has a genetic or familial component. It is believed that the genetic influence occurs through polygenic or multifactorial transmission. Reportedly, 5-50\% of cases of POAG are hereditary, with the best estimate being $20-25 \%$. The risk of developing POAG in first-degree relatives is $4-16 \% .^{3-5}$ The disease has a hereditary component and becomes more prevalent with age.

POAG progresses very slowly and is usually asymptomatic until late in its course, so affected individuals can develop severe damage before they seek professional help. POAG has been shown to be more prevalent in first-degree relatives, so

Correspondence: Dr. Preeti Gupta, 705, Residential Quarters, Institute of Liver and

Biliary Sciences,Vasant Kunj D1, New Delhi-110070, India

E-mail: drpreetigupta82@gmail.com 
their screening for glaucoma is important. Various studies have reported different prevalence depending on the population sampled, the age of the individuals studied, the techniques of examination, and the definitions of glaucoma used.

\section{Methods}

This was a prospective study of first-degree relatives (parents, siblings, and offspring) of patients with POAG and normal tension glaucoma (NTG). Patients with POAG or NTG were requested to give contact details of their first-degree relatives. These first-degree relatives were then contacted and requested to get screening tests for glaucoma done. Informed consent was taken from all the subjects. The study was approved by the hospital ethics committee.

Those with media opacity due to which fundus could not be visualized or perimetry could not be done, or patients with other macular or neurological problems that could affect the test results were excluded from the study.

Subjects underwent detailed ophthalmic examination including visual acuity (using Snellen's chart), slit lamp examination, intraocular pressure (IOP) measurement with Goldmann applanation tonometry, gonioscopy using Goldmann single mirror gonioscope, and fundus examination with a $90 \mathrm{D}$ lens. Pachymetry was done using the Alcon Ocuscan RxP ultrasound pachymeter. In cases where disc findings were suspicious, visual field analysis was done using the Zeiss Humphrey Field Analyser Model 750. When the visual field showed glaucomatous defects, a second field was repeated to confirm the presence of the defects. Glaucomatous disc changes included vertical C:D ratio $>0.6$, focal notch, NRR $<0.1$ in superior or inferior quadrant, disc haemorrhage, nerve fibre layer loss, and cup asymmetry between the two eyes of $>0.2$ when disc size was the same for both eyes. Subjects were labelled as having glaucoma when the field defect met Anderson's criteria for glaucoma diagnosis. Subjects were also asked if they knew the fact that their first-degree relative suffered from glaucoma and the response was noted. Subjects were also asked if they knew that being first-degree relatives of a patient with glaucoma increased their risk of developing glaucoma.

On the basis of test results participants were classified as:

1. Definite glaucoma: glaucomatous disc with corresponding field defect and/ or IOP $\geq 21 \mathrm{mmHg}$.

2. Glaucoma suspects: normal HFA, but IOP $\geq 21 \mathrm{mmHg}$ and/or suspicious, but not unequivocally glaucomatous disc appearance.

3. Normal: normal optic disc, IOP $<21 \mathrm{mmHg}$.

\section{Results}

A total of 66 subjects who satisfied the inclusion and exclusion criteria were included in the study. The age of the subjects ranged from 19-80 years with a 
mean age of $49.6 \pm 12.5$ years. The study population included 38 males $(58 \%)$ and 28 females (42\%). Of the 66 first-degree relatives examined during the study, 23 were siblings (34.8\%) and 43 were offspring (65.2\%). In our study, there were no parents.

Forty-four subjects (66.7\%) were aware of the fact that their first-degree relative had glaucoma and $42(63.6 \%)$ subjects responded negatively when inquired if they knew they were at increased risk for developing glaucoma. Nine subjects were already diagnosed as POAG/NTG and five cases as glaucoma suspects, shown in Table 1. Of the 14 patients classified as glaucoma and glaucoma suspects, 10 (71.4\%) were males and 4 (28.6\%) were females. Seven were siblings and seven were offspring of POAG/NTG patients. Table 2 shows 17 subjects were newly diagnosed as either POAG/NTG (2) or glaucoma suspects (15). Overall, our study categorized a total of 66 subjects, as shown in Table 3.

Thus, in our study we found the prevalence of glaucoma in first-degree relatives to be $16.7 \%$ with an additional $30.3 \%$ subjects as glaucoma suspects. Of the total 11 glaucoma subjects diagnosed, 8 were siblings while 3 were offspring.

Table 1. Subjects already diagnosed as glaucoma and glaucoma suspects

\begin{tabular}{|l|l|l|}
\hline & Frequency & Percentage (\%) \\
\hline Glaucoma suspect & 5 & 35.7 \\
\hline Glaucoma & 9 & 64.3 \\
\hline Total & 14 & 100.0 \\
\hline
\end{tabular}

Table 2. Subjects newly diagnosed as glaucoma and glaucoma suspects

\begin{tabular}{|l|l|l|}
\hline & Frequency & Percentage (\%) \\
\hline Suspect & 15 & 88.2 \\
\hline POAG/NTG & 2 & 11.76 \\
\hline
\end{tabular}

Table 3. Total number of subjects included in the study

\begin{tabular}{|l|l|l|}
\hline & Frequency & Percentage (\%) \\
\hline Normal & 35 & 53.0 \\
\hline Suspect & 20 & 30.3 \\
\hline Glaucoma & 11 & 16.7 \\
\hline Total & 66 & 100.0 \\
\hline
\end{tabular}

\section{Discussion}

Positive family history is an important risk factor for the development of POAG. ${ }^{3,4}$ Early detection of the disease is crucial in slowing down glaucomatous damage 
and minimizing irreversible vision loss, but an effective mass screening program for glaucoma has not yet been developed. One possible strategy for enhancing the effectiveness of glaucoma screening programs is to focus on high-risk populations such as those who have a family history of the disease.

We included all first-degree relatives of patients with a family history of POAG/ NTG willing to participate in our study irrespective of age. In the Glaucoma Inheritance Study in Tasmania, ${ }^{3}$ all the relatives were included in the study, with age ranging from 13 to 97 years. We included all first-degree relatives who were willing to come to the hospital for examination in our study. Age range in our study was 19-80 years. Other studies set a lower age limit for subjects to be included: in Vegini et $a l .{ }^{5}$ the lower limit was 30 years; in Nguyen et al. ${ }^{6}$ the lower limit was 35 years; and in the Beaver Dam Eye study the lower limit was 43 years. ${ }^{7}$ We included all first-degree relatives in our study, which may affect the study outcome, as young subjects are less likely to develop glaucoma and therefore project a lower prevalence.

The average age of the study population was 49.6 years (S.D 12.5) with a median of 48.5 years, similar to studies done by Vegini et al. ${ }^{5}$ and Nguyen et al., ${ }^{6}$ but lower than many other studies. ${ }^{3,4,8}$ Our study included $34.8 \%$ siblings and $65.2 \%$ offspring.

The Glaucoma Inheritance Study in Tasmania ${ }^{5}$ and other states in Australia examined 442 individuals from five pedigrees with a strong positive family history of POAG (not only first-degree relatives), of which $11 \%$ had a prior diagnosis of POAG, $2 \%$ had glaucoma-suspect status, and $16 \%$ were newly diagnosed $(7 \%$ POAG and $9 \%$ suspects). Prevalence rate in first-degree relatives was $38.6 \%$. We found a prevalence rate of glaucoma of $16.7 \%$ with $13.6 \%$ previously diagnosed and $3 \%$ as newly diagnosed cases. This variation in the prevalence may be due to the fact that the Glaucoma Inheritance study in Tasmania was a broad-based study with a large subject size, so most of the first-degree relatives were included in the study.

The Rotterdam Eye Study ${ }^{8}$ investigated the familial aggregation of POAG by examining the first-degree relatives (siblings and children) of 45 of the 48 cases of glaucoma identified, as well as a matched set of controls. The prevalence of glaucoma was $10.4 \%$ in siblings of patients and $1.1 \%$ in offspring of patients. This is similar to our study, where we found a prevalence rate of $12.1 \%$ in siblings of patients and $4.5 \%$ in offspring of patients.

The study by Nguyen et al. ${ }^{6}$ examined 86 relatives from 15 families with chronic open-angle glaucoma (OAG), including 50 first-degree relatives. In these 50 first-degree relatives, 11 siblings (22\%), 4 children (8\%), and $3(6 \%)$ parents were diagnosed with glaucoma, for a total glaucoma prevalence rate of $36 \%$ in first-degree relatives. The difference in the results may be due to the fact that the study 
done by Nguyen tried to include most of the relatives in these families, while in our study not all first-degree relatives could be included in the study.

The Barbados Family Study ${ }^{4}$ investigated the potential role of inheritance of OAG among families of African origin. A total number of 1056 relatives were included in the study, of which 865 were first-degree relatives (21 parents, 181 full sibling, 157 half sibling, and 506 offspring). Prevalence rates among them was $0.9 \%$ for parents, $4.5 \%$ for full sibling, $3.23 \%$ for half sibling, and $1.3 \%$ for offspring. The difference in our study results could be due to the fact that not all first-degree relatives were included in the study; racial factors might also play a role.

The study by Vegini et $a .^{5}$ examined 101 first-degree relatives accompanying POAG patients to the hospital. They found the prevalence rate of glaucoma among these first-degree relatives to be $16.8 \%$, with $10.9 \%$ previously diagnosed cases and $5.9 \%$ freshly diagnosed cases. This result is similar to our study, where we found a prevalence rate of $16.6 \%$, with $13.6 \%$ previously diagnosed cases and $3 \%$ newly diagnosed cases.

In a study by Kong et al. ${ }^{9}$ of 531 first-degree relatives screened, 67 (12.62\%) were identified to have POAG, a rate eight times higher than that of the control group ( 8 of 526, 1.52\%). The effect of family history on parents, siblings, and offspring of probands was statistically significant, with OR values of 6.92 ( $95 \% \mathrm{Cl}: 1.90-25.18)$, 11.29 (95\% Cl: 3.63-35.11), and 11.35 (95\% Cl: 1.69-76.21), respectively. In the case of glaucoma suspects, a significant effect was found for both family units (OR 5.60; 95\% Cl: 1.15-27.21) and offspring (10.83 OR; 95\% Cl: 1.34-87.73).

In a south Indian study by Rajendrababu et al. ${ }^{10}$ of 514 first-degree relatives of POAG patients examined, 68 (13.3\%) had definite glaucoma while another 28 $(5.5 \%)$ were either ocular hypertension or glaucoma suspects. This result shows nearly similar prevalence rates to our study.

Limitations of our study include the small sample size and the fact that we could not include all first-degree relatives of glaucoma patients, therefore the results might not reflect the actual prevalence. The reasons why not all first-degree relatives could be included were: migration of relatives to different cities, inability to come to the hospital for examination, and lack of interest for the examination. This again projects the difficulty in screening all first-degree relatives of glaucoma patients. Furthermore, we compared our results mostly with non-Asian countries, which may also account for a difference in prevalence rates.

Our study found that $66.7 \%$ of the subjects were aware of the family history of glaucoma, but only $36.4 \%$ were aware that they were at increased risk of developing glaucoma. In a study by Friedman et al. ${ }^{11}$ among 102 probands and 100 (of 230 eligible) family members who were interviewed, $21 \%$ of both groups were not aware that OAG is hereditary. This shows the lack of awareness about increased hereditary risk of glaucoma among the general population. Although the subjects 
were aware of the fact that their relative had glaucoma, they did not know that they were at increased risk and needed to undergo glaucoma screening.

Our study emphasizes the need for screening first-degree relatives of patients with POAG/ NTG. Selective screening of first-degree relatives of POAG/NTG patients helps in early disease diagnosis. We also need a large population-based study to find out the prevalence rates of glaucoma in relatives of glaucoma patients among the Indian population.

\section{References}

1. World Health Organization. Global data on visual impairments 2010. Available from: http://www. who.int/blindness/GLOBALDATAFINALforweb.pdf.

2. Ramakrishnan R, Nirmalan PK, Krishnadas R, et al. Glaucoma in a rural population of Southern India: The Aravind Comprehensive Eye Survey. Ophthalmology. 2003;110:1484-1490. doi: 10.1016/S0161-6420(03)00564-5.

3. McNaught Al, Allen JG, Healey DL, et al. Accuracy and Implications of a Reported Family History of Glaucoma: Experience From the Glaucoma Inheritance Study in Tasmania. Arch Ophthalmol. 2000;118(7):900-904.

4. Leske MC, Nemesure B, He Q, Wu SY, Fielding Hejtmancik J, Hennis A. Patterns of open-angle glaucoma in the Barbados Family Study. Ophthalmology. 2001;108(6):1015-1022.

5. Vegini F, Figueiroa Filho N, Lenci RF, Garcia Neto D, Susanna Junior R. prevalence of open angle glaucoma in accompanying first degree relatives of patients with glaucoma. Clinics (Sao Paulo) 2008;63(3):329-332 doi: 10.15900/S1807-59322008000300007.

6. Nguyen RL, Raja SC, Traboulsi El. Screening relatives of patients with familial chronic open angle glaucoma. Ophthalmology. 2000;107(7):1294-1297.

7. Klein BE, Klein R, Sponsel WE, et al. Prevalence of glaucoma: The Beaver Dam Eye Study. Ophthalmology. 1992;99(10):1499-1504

8. Wolfs RC, Klaver CC, Ramrattan RS, van Duijn CM, Hofman A, de Jong PT. Genetic risk of primary open-angle glaucoma. Population-based familial aggregation study, Arch Ophthalmol. 1998;116(12):1640-1645.

9. Kong X, Zhu W, Chen X, Chen Y, Sun X. Familial aggregation of primary open angle glaucoma in Shanghai, China. Mol Vis. 2013;19:1859-1865.

10. Rajendrababu S, Gupta N, Vijaykumar B, Kumaragurupari R, Krishnadas SR. Screening first degree relatives of persons with primary open angle glaucoma in India. J Curr Glaucoma Pract. 2014;8(3):107-112. doi:10.5005/jp-journals-10008-1172.

11. Okeke CN, Friedman DS, Jampel HD, et al. Targeting Relatives of Patients With Primary Open Angle Glaucoma: The Help the Family Glaucoma Project. J Glaucoma. 2007;16(6):549-555. doi: 10.1097/IJG.0b013e3180391a4c. 